\title{
Alterstice
}

Revue internationale de la recherche interculturelle

International Journal of Intercultural Research

Revista International de la Investigacion Intercultural

\section{O Psicólogo frente ao desafio tecnológico: Novas identidades, novos campos, novas práticas, sous la direction de Elaine Magallhães Costa Fernandez et Véronique Donard}

\section{Candy Marques Laurendon}

Volume 7, numéro 2, 2017

URI : https://id.erudit.org/iderudit/1052574ar

DOI : https://doi.org/10.7202/1052574ar

Aller au sommaire du numéro

Éditeur(s)

Alterstice

ISSN

1923-919X (numérique)

Découvrir la revue

Citer ce compte rendu

Marques Laurendon, C. (2017). Compte rendu de [O Psicólogo frente ao desafio tecnológico: Novas identidades, novos campos, novas práticas, sous la direction de Elaine Magalhães Costa Fernandez et Véronique Donard]. Alterstice, 7(2),

117-120. https://doi.org/10.7202/1052574ar d'utilisation que vous pouvez consulter en ligne. 
NOTE DE LECTURE

\title{
O Psicólogo frente ao desafio tecnológico: Novas identidades, novos campos, novas práticas, sous la direction de Elaine Magalhães Costa Fernandez et Véronique Donard
}

Candy Marques Laurendon ${ }^{1}$

\author{
Rattachement de l'auteure \\ ${ }^{1}$ Université Fédérale de Pernambuco (UFPE), Brésil et Laboratoire Clinique, Psychopathologie interculturelle (LCPI) \\ de l'Université Toulouse Jean-Jaurès, France
}

\section{Correspondance}

nacyd@hotmail.fr

\section{Références de l'ouvrage}

Costa Fernandez, E. M. et Donard, V. (dir.) (2016). O Psicólogo frente ao desafio tecnológico: Novas identidades, novos campos, novas práticas. Recife, Brésil: Editions UFPE et UNICAP.

\section{Pour citer cet article}

Marques Laurendon, C. (2016). O Psicólogo frente ao desafio tecnológico: Novas identidades, novos campos, novas práticas [Note de lecture]. Alterstice, 7(2), 117-120.

Cet ouvrage, d'une grande actualité scientifique, soulève des questions innovantes sur le rôle du psychologue et de la psychologie dans la compréhension des répercussions des Nouvelles Technologies de I'Information et de la Communication (NTIC) dans la contemporanéité. Au delà de l'analyse de quelques pratiques professionnelles en lien avec ces nouvelles pratiques culturelles, les textes réunis dans cet ouvrage collectif proposent des pistes de recherche tant conceptuelles que méthodologiques, nécessaires à la construction d'un nouveau champ de savoir. Les organisatrices, psychologues bilingues avec une grande expérience professionnelle et académique internationale proposent une œuvre collective bilingue originale, avec des résumés en français pour les textes en portugais et des résumés en portugais pour les textes en français.

Il ressort des quatorze chapitres rédigés par des experts internationaux, enseignants chercheurs pour la plupart, que l'utilisation massive des NTIC, symbole de la modialisation, engendre des conséquences sur le psychisme des usagers, comme l'émergence de nouvelles identités, de nouvelles modalités de subjectivation et d'interactions sociales. Par ailleurs, les professionnels en santé publique et les chercheurs en sciences humaines et sociales sont appelés à concevoir des nouvelles modalités d'intervention, notamment pour le dépistage et le soin des abus de ces artefacts de la mondialisation. Ces questions interrogent donc particulièrement les psychologues interculturels, 
amenés à innover avec des interventions professionnelles efficaces et des politiques publiques qui prennent en compte l'appartenance culturelle des usagers. La lecture de ces textes favorise la compréhension de phénomènes humains comme la démocratisation en lien avec les NTIC et des alternatives de résolution des problèmes engendrés par l'impact de ces technologies sur les sujets et les sociétés actuelles.

Le livre est composé de deux parties. La première réunit sept textes sur les Nouvelles Technologies de I'Information et de la Communication (NTIC) et la recherche en psychologie.

Le premier texte, d’Elaine Magalhães Costa Fernandez, traite de la possible contribution des NTIC, « symbole hégémonique de la globalisation ", à la promotion du processus de démocratisation et à la reconnaissance de la diversité culturelle, interprétées comme des pratiques qui répondent le mieux aux attentes de la société actuelle. En s'appuyant sur des auteurs contemporains brésiliens et internationaux, l'auteure propose une discussion critique pour adopter une approche positive des nouvelles technologies en montrant la nécessité de l'application de politiques publiques pour stimuler l'inclusion sociale et l'exercice de la citoyenneté par les usagers à travers l'adoption de certaines valeurs comme le respect de l'altérité et de l'individualité de chacun.

Le deuxième texte, de Véronique Donard, propose de caractériser les fondements épistémologiques et les nouveaux paradigmes d'une révolution technologique existentielle. L'essor des NTIC révèle l'existence d'une révolution technologique qui est devenue une véritable "révolution existentielle », en modifiant les vécus et les perceptions des sujets, notamment à travers une dimension espace-temps différente, possible grâce à l'utilisation de ces technologies. Plus qu'un simple simulateur de la réalité, le numérique devient créateur d'une nouvelle réalité qui a un impact sur le sujet. Après avoir discuté les concepts de "virtuel " et de "cyber» en approfondissant la nature de la cyberpsychologie, l'auteur termine en caractérisant le terme " digital » traduit par " numérique » et ses multiples usages, et invite à la création et à la multiplication des recherches dans ce domaine.

Dans le troisième texte, intitulé "Panorama et enjeux des mondes numériques », Benoit Virole propose, à partir de la description de la mutation anthropologique provoquée par l'implantation des technologies numériques, quelques opérateurs conceptuels qui permettent de mieux comprendre le phénomène et le processus psychologique d'adhésion à une réalité virtuelle pour le sujet. L'auteur montre ainsi comment le monde numérique transforme les processus de production et de communication des connaissances et modifie les rapports sociaux, les parents déléguant leurs fonctions de protection à des systèmes automatiques externes par exemple. La culture numérique provoque également une dégradation de certaines valeurs comme l'amitié, en confondant une relation numérique avec « un phénomène psychique, social et complexe ».

Le quatrième texte, de Julien Teyssier et Patrick Denoux, concerne les réactions psychologiques transitoires et interculturations face aux ruptures culturelles provoquées par l'émergence des artefacts numériques, qualifiées de "véritable rupture anthropologique ". Après avoir décrit l'incidence des changements dans l'environnement culturel causés par l'ère numérique, les effets de cette rupture anthropologique sont illustrés à partir des différentes réactions psychologiques transitoires entre deux populations : les " digital natives » et les « digital immigrants ». Ainsi, contrairement au migrant numérique qui réussit à créer de façon harmonieuse ou non une culture tierce, l'hétérogeneité culturelle d'expériences provoque, chez le natif numérique, de multiples réactions psychologiques transitoires, démontrant un processus d'interculturation très singulier, lorsqu'il existe.

Dans le cinquième texte, intitulé "Contrôle d'informations sociales dans les environnements numériques: répercurssions sur la construction de narratives de soi ", José Carlos Ribeiro traite de la gestion et du contrôle, à travers la publication et la divulgation d'informations, des comportements expressifs dans ces environnements médiatisés complexes de communication. L'auteur montre que le sujet autorégule ces informations sociales de soi, en prenant en compte la situation sociale et ses facteurs ainsi que les attentes de l'audience pour (re)construire des discours, mémoires et narrations personnelles davantage proches d'images idéalisées, ce qui contribue à l'émergence d'un self constitué.

Le texte suivant, de Viviane de Bona, porte sur les nouvelles conceptions à propos du fait d'« être enfant» aujourd'hui dans une société marquée par les technologies. L'auteur propose une discussion sur l'impact des 
technologies dans le développement infantile, tantôt perçu comme négatif car rapprochant dangereusement les enfants du monde adulte, tantôt positif car contribuant à la constitution d'une génération actrice d'une possible transformation de la société. La recherche présentée dans ce texte, réalisée avec 52 enfants, révèle que les technologies sont incorporées dans leur quotidien infantile en raison de leur attractivité et pour constituer une nouvelle forme de loisirs et une manière de communication sociale avec leurs pairs.

De la même façon, le texte suivant, de Lucia de Mello e Souza Lehmann et Artur Parreira, intitulé "Contextes virtuels: univers d'interaction de jeunes ", traite des différents modes d'appropriation et d'utilisation des médias, cette fois-ci par des jeunes de milieu universitaire. Après avoir analysé l'impact des nouvelles technologies sur le plan cognitif, affectif et relationnel, en particulier sur les modes de vivre et d'apprendre, les auteurs proposent de comparer les résultats de recherches sur les réseaux groupaux avant l'apparition des nouvelles technologies, c'està-dire les cultures juvéniles dans leurs anciens contextes physiques et actuellement dans les contextes virtuels. Les nouvelles transformations dans les modes de vie et d'apprentissage des jeunes en lien avec les technologies posent des défis autant pour les jeunes que pour les adultes.

La seconde partie du livre réunit sept textes autour de la thématique des Nouvelles Technologies de l'Information et de la Communication (NTIC) et des interventions psychologiques et des politiques publiques.

Tout d'abord, Odette Lescarret nous invite à une réflexion sur les défis de la communication par Skype pour des enfants de 3 ans et moins et leurs jeunes parents dans deux contextes socioculturels différents. Cette période de la première enfance est caractérisée par l'expérience proprio-perceptive et la manipulation d'objets concrets comme la nécessité de contact corporels, notamment avec les parents, pour permettre la constitution de l'image corporelle. À partir de trois cas cliniques, l'auteure pose la question de l'impact de la relation virtuelle, établie entre parent et enfant, sur la privation d'expériences sensori-motrices et les différences dans la voix transmise par ce média. Elle conclut en suggérant de nouvelles pistes au psychologue pour guider les parents et grands-parents «Skype » et éviter les conséquences de cette privation.

Les trois textes suivants traitent de l'utilisation de divers recours technologiques dans le cadre de la psychothérapie. Edilene Freire de Queiroz et Véronique Donard évoquent divers constituants des thérapies en ligne concernant ce nouveau setting psychanalytique qui interroge les dimensions espace-temps et la perception sensorielle, notamment à travers l'importance du regard. Yann Leroux démontre l'utilité des jeux vidéo comme médiateurs pour permettre d'établir une relation de travail stable et positive entre le thérapeute et le jeune et ainsi identifier les conflits et les anxiétés. L'auteur recommande alors au thérapeute d'abandonner ses préconceptions à l'égard de ces instruments qui se présentent comme facilitateurs de la relation thérapeutique. Le quatrième texte, de Leopoldo Barbosa, analyse l'apport de l'utilisation des ressources technologiques et des jeux électroniques dans la psychothérapie, en considérant différentes spécialités psychologiques comme la neuropsychologie et les réhabilitations des processus cognitifs déficitaires, les thérapies cognitivo-comportementales et la psychologie positive.

Le cinquième texte, de Charlotte Soumet-Leman, aborde également l'intérêt de l'utilisation de l'ordinateur pendant le processus de réhabilitation cognitive, appropriée pour les personnes souffrant de dépression et manifestant des troubles cognitifs. À partir d'une étude de cas, sont révélés les effets de l'utilisation d'un dispositif pour l'accompagnement en Réhabilitation Cognitive Assistée par Ordinateur (RCAO), notamment le fait qu'ils favorisent une meilleure réactivité et une flexibilité mentale pour le sujet.

Les deux derniers textes du livre abordent les politiques publiques sur l'insertion des NTIC dans le milieu éducatif de l'État de Pernambuco. Prenant l'exemple de dispositifs mobiles comme les smartphones, interdits dans les salles de classe par la Loi de 2015, Marina Pinheiro et Luciano Meira analysent l'adhésion des jeunes aux applications téléchargées et aux réseaux sociaux. Ceci devrait être l'objectif de l'éducation : proposer des environnements qui stimulent la créativité, l’imagination et l'expérimentation immersive. Car la technologie peut être entendue « comme un appui amplificateur des changements culturels, relationnels et cognitifs autant au niveau politique que pédagogique ", favorisant dans le milieu éducatif de nouvelles conditions dialogiques, esthétiques et immersives du contexte d'apprentissage et la non-évasion des significations des étudiants dans les systèmes d'enseignement. De 
même, le dernier texte de Viviane de Bona et Dayse Rodrigues de Oliveira aborde les politiques publiques d'inclusion numérique dans les écoles. Les auteurs présentent une recherche sur l'étude de ces politiques publiques et leurs réelles applications dans le milieu scolaire, toujours dans l'État de Pernambuco. Les résultats révèlent certains paradoxes dans cette application comme le manque d'infrastructures adéquates et la faible orientation pour l'utilisation consciente de ces technologies, qui ne contribuent pas à une véritable inclusion numérique dans les écoles, observée de façon superficielle dans les pratiques des étudiants. Les auteurs concluent en recommandant de penser les politiques publiques comme une possibilité de transformation sociale des élèves des écoles publiques brésiliennes.

Ces différents textes, avec des aspects tant théoriques que pratiques, permettent au lecteur de revenir sur sa propre compréhension du rôle joué par les NTIC sur les usagers et la société en général. La variété des points de vue ainsi que la diversité culturelle des auteurs contribuent à l'appréhension de la complexité du phénomène, en révélant ce qui se fait déjà mais également ce qui reste à faire à l'école, en famille, dans les tribunaux ou dans le monde du travail. Des perspectives sont donc ouvertes en matière de recherche, de pratiques professionnelles et de politiques publiques. Dans un monde sujet à une évolution de plus en plus rapide, où la mobilité devient une valeur recherchée, cet ouvrage rassure le lecteur par ses arguments et les analyses proposées. Je vous conseille donc sa lecture pour la pertinence, la qualité et la richesse de son contenu. 